\title{
Etnoecologia comportamental da baleia-franca-austral Eubalaena australis (Desmoulins, 1822) por meio do conhecimento ecológico local de pescadores artesanais na Área de Proteção Ambiental da Baleia Franca, Sul do Brasil Ethnoecology behavioral of Southern Right Whale Eubalaena australis (Desmoulins, 1822) through the local ecological knowledge of artisanal fishermen in Southern Right Whale Area of Environmental Protection, southern Brazil
}

\author{
Camilah Antunes Zappes", "I (D) | Mônica Lauriano Danielskill (D) | Ana Paula Madeira Di Beneditto "I, III (D) \\ Universidade Federal Fluminense. Programa de Pós-Graduação em Geografia. Rio de Janeiro, Rio de Janeiro, Brasil \\ "Universidade Federal Fluminense. Grupo de Pesquisa em Ecologia Humana e Conservação de Recursos Culturais e Naturais. \\ Rio de Janeiro, Rio de Janeiro, Brasil \\ "'Iniversidade Estadual do Norte Fluminense. Programa de Pós-Graduação em Ecologia e Recursos Naturais. \\ Rio de Janeiro, Rio de Janeiro, Brasil
}

\begin{abstract}
Resumo: $\bigcirc$ objetivo deste estudo foi caracterizar o conhecimento ecológico local dos pescadores artesanais do município de Garopaba, Santa Catarina, Sul do Brasil, em relação ao comportamento da baleia-franca-austral, Eubalaena australis, na Área de Proteção Ambiental (APA) da Baleia Franca. Em outubro de 2010, foram realizadas 33 entrevistas etnográficas com pescadores em que 81,8\% ( $n=27)$ dos entrevistados identificam a espécie por meio da sua área de ocorrência, coloração e tamanho corporal. As análises das entrevistas foram baseadas nestes 27 pescadores selecionados, com a descrição de mais de uma etnocategoria por entrevistado. Os pescadores indicaram dez etnocategorias comportamentais comuns a adultos e infantes, e a categoria 'não faz nada' frente a embarcações ( $n=13 ; 26,0 \%)$ foi a mais frequente. Para comportamentos específicos de adultos, foram descritas 25 etnocategorias e a mais frequente foi 'brincar' ( $n=13$; 18,1\%). Quatro etnocategorias foram descritas para infantes $(n=18)$ e estão relacionadas à alimentação/amamentação $(n=15 ; 83,3 \%)$ e à vocalização/eventos de interação com a fêmea adulta $(n=3 ; 16,7 \%)$. A partir das experiências empíricas proporcionadas pela prática pesqueira artesanal, os entrevistados foram capazes de reconhecer a espécie, distinguir indivíduos adultos e infantes e descrever suas principais características comportamentais.
\end{abstract}

Palavras-chave: Cetáceos. Comportamento. Conhecimento ecológico local. Estado de Santa Catarina.

Abstract: The aim of this study is to characterize the local knowledge displayed by the fishermen at Garopaba Municipality, Santa Catarina state, Brazil, regarding the behavior of Southern Right Whale, Eubalaena australis, in the region of the Southern Right Whale Area of Environmental Protection (AEP). In October of 2010 there were performed 33 interviews with local fishermen and found that $81.8 \%(n=27)$ of the interviewed were able to identify the species by its area of occurrence, coloration, and body size. The analyses were based on interviews of 27 selected fishermen. Were described 10 common behavioral ethnocategories adults and infants, in which the most frequent was 'do nothing' to vessels ( $n=13 ; 26.0 \%)$. For the specific behaviors of the adult were described 25 ethnocategories $(n=72)$ and the most frequent was 'playing' $(n=13,18.1 \%)$. Four ethnocategories been described for infants $(n=18)$ and are relate to feeding/breastfeeding $(n=15,83.3 \%)$ and the calling/ interaction events with the adult female $(n=3,16.7 \%)$. From the empirical offered by artisanal fishing, the interviewed were able to recognize the species, to distinguish between adults and infants and to describe its main behavioral characteristics.

Keywords: Cetacean. Behavior. Local traditional knowledge. Santa Catarina state.

ZAPPES, C. A., M. L. DANIELSKI \& A. P. M. DI BENEDITTO, 2020. Etnoecologia comportamental da baleia-franca-austral Eubalaena australis (Desmoulins, 1822) por meio do conhecimento ecológico local de pescadores artesanais na Área de Proteção Ambiental da Baleia Franca, Sul do Brasil. Boletim do Museu Paraense Emílio Goeldi. Ciências Naturais 15(3): 601-616. DOI: http://doi.org/10.46357/ bcnaturais.v15i3.183.

Autora para correspondência: Camilah Antunes Zappes. Rua José do Patrocínio, 71 - Centro. Campos dos Goytacazes, RJ, Brasil. CEP 28010-385 (camilahaz@yahoo.com.br).

Recebido em 05/09/2019

Aprovado em 08/09/2020

Responsabilidade editorial: Alexandra Maria Ramos Bezerra
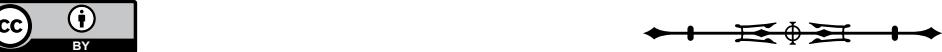


\section{INTRODUÇÃO}

A Etnociência é o estudo do conhecimento de populações humanas sobre o mundo natural, as taxonomias e classificações. Esta ciência descreve práticas e crenças de comunidades tradicionais e pode, ainda, realizar comparações entre o conhecimento praticado pelas comunidades humanas e o descrito pelo meio acadêmico (Farias \& Alves, 2007; Roué, 2000). O termo Etnoecologia permite a integração entre o saber acadêmico e o saber social na área da Ecologia, já que é uma ciência voltada para o estudo do conhecimento dos grupos tradicionais, bem como suas práticas e crenças em relação ao ecossistema (Diegues, 2000). A partir dessa proposta, pesquisadores que estudam a Etnoecologia trabalham com pescadores artesanais, a fim de compreender o modo como estes atores se relacionam com o ambiente (Diegues, 2000).

Pescadores artesanais são definidos como um grupo tradicional que utiliza a mão de obra familiar durante a prática da pesca e cuja subsistência é baseada nesta atividade (Diegues, 2002). Essas comunidades apresentam vasto conhecimento pesqueiro empírico, já que exploram hábitats costeiros e de águas interiores há várias gerações. Esses atores locais, por meio de suas observações, são capazes de fornecer informações relevantes sobre a biologia e a ecologia das espécies que se distribuem nos campos de pesca (Costa-Neto, 2000). O conhecimento ecológico local (CEL) pode ser definido como aquele adquirido por uma comunidade por meio do contato do homem com o ambiente, sendo transmitido ao longo de gerações (Berkes, 1999; Clauzet et al., 2005).

No município de Garopaba, litoral do estado de Santa Catarina (SC), Sul do Brasil, a pesca artesanal é importante na economia das populações tradicionais. Parte deste município está inserida na Área de Proteção Ambiental (APA) da Baleia Franca e há sobreposição sazonal de áreas de uso entre os pescadores artesanais e as baleias-francas-austrais Eubalaena australis (Desmoulins, 1822) (IWC/Brasil, 1999). Essa espécie se distribui no Hemisfério Sul, entre $20^{\circ} \mathrm{S}$ e $64^{\circ} \mathrm{S}$ (IWC/Brasil, 1999; Jefferson et al., 2015), e na região de Garopaba é registrada uma população semirresidente nos meses de inverno e na primavera (julho a novembro). Neste período, as baleias migram para o litoral da América do Sul para o nascimento e a amamentação dos filhotes, assim como para a atividade reprodutiva (Danielski, 2008; Groch, 2000; Jefferson et al., 2015). Nos últimos quinze anos, o número de baleias que migram sazonalmente para o litoral do país tem aumentado a cada ano (Groch et al., 2005).

Interações entre a pesca artesanal e a baleia-francaaustral são descritas por pescadores, as quais indicam conflitos entre a espécie e a atividade pesqueira, como colisões com embarcações e captura acidental por redes de pesca, que causam ferimentos aos animais e danos aos petrechos de pesca (Zappes et al., 2013a, 2013b). Ainda, a cada estação de migração da baleia-franca-austral para a costa brasileira, aumentam as avistagens desses organismos pelos pescadores, já que as rotas das baleias coincidem com as principais áreas de pesca artesanal (Bueloni, 2012; Zappes et al., 2013a, 2013b).

Apesar destas informações, estudos relacionados ao conhecimento ecológico local de pescadores artesanais sobre baleias são escassos e ainda incipientes no litoral brasileiro (Bueloni, 2012; Zappes et al., 2013a, 2013b), o que indica a necessidade da realização de trabalhos que envolvam esta questão. Compreender a etnoecologia de cetáceos marinhos pode facilitar o diálogo e a integração entre os conhecimentos tradicional e científico e, desta forma, aplicar medidas eficazes para a conservação dessas espécies, por meio da cogestão da pesca artesanal (Huntington et al., 2002; Zappes et al., 2016).

O presente estudo descreve, pela primeira vez para a espécie, o etnoconhecimento dos pescadores artesanais sobre os comportamentos exibidos pela baleia-francaaustral na APA da Baleia Franca e compara as informações obtidas com dados da literatura, de modo a avaliar o conhecimento ecológico local desta comunidade. 


\section{MATERIAL E MÉTODOS}

\section{ÁREA DE ESTUDO}

O município de Garopaba (28 01' S, 48 36' O), localizado no litoral do estado de Santa Catarina (SC), Brasil, apresenta área costeira de 108 km² e está inserido na APA da Baleia Franca (Figura 1). Nesta região está sediada a Colônia de Pescadores Z-12, que reúne 11 núcleos pesqueiros em atividade (Rebouças, 2008). Os núcleos são frequentados por pescadores das comunidades de Garopaba e dos municípios vizinhos, com 1.500 pescadores cadastrados (Capellesso \& Cazella, 2011), sendo que nas comunidades da Praia de Gamboa existem 30 pescadores registrados, e da Praia de Garopaba, 50 registrados.

\section{PROCEDIMENTOS}

As informações foram obtidas em outubro de 2010, a partir de 33 entrevistas etnográficas realizadas nas comunidades de Gamboa ( $n=14$ ) e Garopaba ( $n$ $=19$ ). Estudos relacionados à Etnociência indicam que o tamanho ideal de amostragem varia de 30 a 60 entrevistas (Bernard, 2000; Mason, 2010; Morse, 1994). O número de entrevistas aplicadas no presente trabalho justifica-se pelo fato de que, em cada embarcação, trabalham de dois a quatro pescadores, os quais podem atuar em mais de uma embarcação; além disso, a partir da décima entrevista houve o mesmo padrão de resposta entre os pescadores (ponto de saturação) (Thiry-Cherques, 2009).

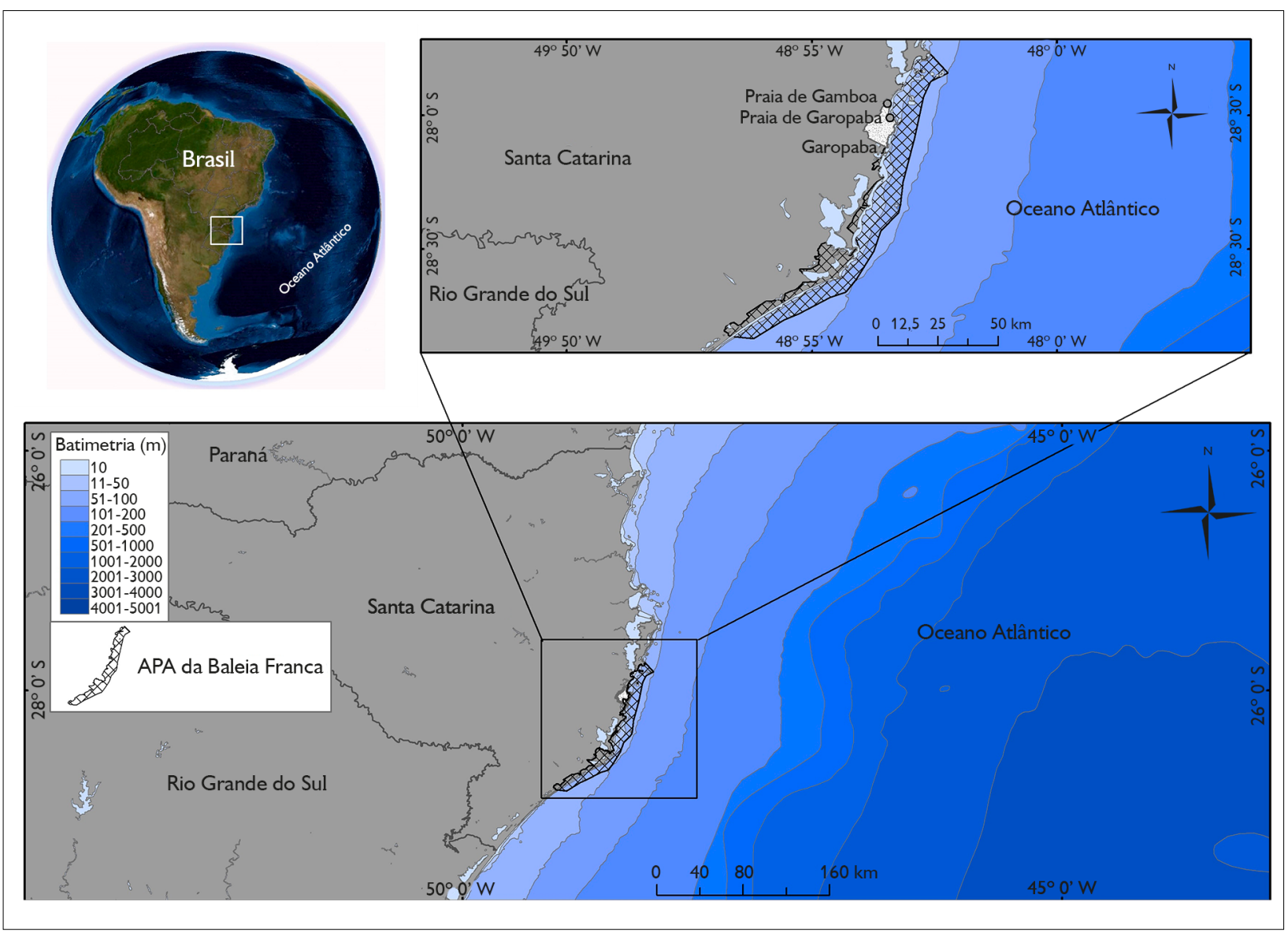

Figura 1. Localização da Área de Preservação Ambiental (APA) da Baleia Franca e do município de Garopaba (área delimitada em branco no mapa menor, detalhado), estado de Santa Catarina, Sul do Brasil. Mapa: Sérgio Carvalho Moreira (2019).

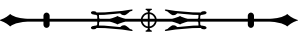


As entrevistas foram orientadas por um questionáriopadrão previamente elaborado (Schensul et al., 1999) com questões semiestruturadas abertas $(n=49)$ e fechadas $(n=19)$. Antes de cada entrevista, foram explicados os objetivos do estudo e fornecida a garantia de preservação da identidade a cada entrevistado (Librett \& Perrone, 2010). Apenas o primeiro nome do pescador era solicitado pelo pesquisador, de modo a facilitar a comunicação. $O$ projeto também foi cadastrado no Sistema de Gestão Nacional do Patrimônio Genético e do Conhecimento Tradicional Associado (SISGEN) (número do cadastro AE2CAEC) e recebeu anuência para sua realização do representante legal dos entrevistados, que é o presidente da Colônias de Pescadores Z-12 (Brasil, 2015).

Todas as entrevistas foram conduzidas por meio de diálogos (entrevista 'pessoal' ou 'face a face'), em formato de pergunta-resposta, a fim de gerar confiança entre entrevistador e entrevistado e aumentar a confiabilidade dos dados (Zappes et al., 2011a). O estabelecimento de diálogo entre pesquisador e entrevistado garante a liberdade do informante durante os relatos e desenvolve uma visão compartilhada da realidade local (Kvale, 1996). questionário foi dividido nas seguintes categorias: (1) perfil dos entrevistados (sexo, idade, período de atuação na pesca); (2) descrição da atividade pesqueira (tecnologia dos artefatos, embarcações e espéciesalvo) (Begossi, 2001); (3) e características morfológicas e comportamentais da espécie em estudo, no caso a baleia-franca-austral $E$. australis (Tabela 1). Inicialmente, as perguntas se relacionaram às baleias adultas e, posteriormente, aos indivíduos infantes (neonatos e juvenis). A fim de distinguir indivíduos adultos de infantes, os pescadores informaram que 'adulto' apresentava maior tamanho corporal quando comparado ao infante, além de descreverem que 'infantes' não apresentam todas as calosidades no dorso. Deste modo, foi possível que os entrevistados descrevessem com clareza cada uma das duas faixas etárias abordadas. Algumas perguntas tiveram respostas fechadas, seguidas de uma justificativa ou explicação aberta para que o entrevistado pudesse expor o seu raciocínio.

Para o primeiro contato com o presidente da Colônia de Pescadores Z-12, foi utilizada uma agente intermediária, que desempenhou a função de guia durante o trabalho de campo (Santos, 2006). A seleção do primeiro entrevistado ocorreu por meio do auxílio do presidente da instituição de pesca, já que, geralmente, é aquele que melhor conhece os demais (Sanches, 2004). O método bola-de-neve, ou método de rede, foi empregado a partir da segunda entrevista, em que foi solicitado a cada entrevistado que indicasse outro potencial entrevistado (Bailey, 1994; Biernacki \& Waldorf, 1981). Ainda, era aplicada a aleatoriedade, por meio de encontros oportunísticos com os pescadores (Paes \& Zappes, 2016).

Os termos utilizados no questionário (Anexo 1) estavam em conformidade com o vocabulário usual dos entrevistados, a fim de evitar dúvida sobre as questões formuladas. A equipe se apresentou como membro de instituição de ensino e pesquisa, para não haver associação com autoridades ou órgãos de fiscalização. Todos os pescadores entrevistados foram escolhidos por meio dos seguintes critérios: (1) ser pescador artesanal; (2) ter a pesca

Tabela 1. Tópicos das questões abordadas no questionário semiestruturado.

\begin{tabular}{c} 
1. Perfil dos entrevistados \\
Sexo \\
Idade \\
Período de atuação na pesca \\
\hline 2. Descrição da atividade pesqueira artesanal \\
\hline Tecnologia dos artefatos \\
Embarcações (comprimento e potência do motor) \\
Espécies-alvo \\
\hline 3. Características de Eubalaena australis \\
Comprimento corporal \\
Coloração \\
Área de ocorrência \\
Ecologia comportamental
\end{tabular}

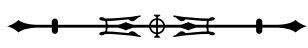


como principal atividade econômica; (3) praticar a pesca artesanal na APA da Baleia Franca. Para evitar a interferência de um informante sobre os outros, cada entrevista foi conduzida em caráter individual (Zappes et al., 2013a, 2013b). Ao final de cada entrevista, foi apresentada ao entrevistado uma prancha ilustrativa (Anexo 2) contendo fotos de espécies de cetáceos com registro na região e que são classificadas pela cultura popular como 'baleias' [A - orca (Orcinus orca Linnaeus, 1758), B - baleia-francaaustral (E. australis), C - baleia-de-bryde (Balaenoptera edeni Anderson, 1879), D - baleia-jubarte (Megaptera novaeangliae Borowski, 1871)]. A estimulação visual ajuda na análise dos relatos e facilita a identificação das espécies pelos entrevistados (Miranda et al., 2007).

Após as entrevistas, as informações foram agrupadas por categorias de temas, para esclarecer a relação entre a linguagem e a interação social, por meio da aplicação da análise do discurso para o desenvolvimento da percepção dos pescadores sobre os comportamentos da baleiafranca-austral (Rocha \& Deusdará, 2005). A certeza do reconhecimento da espécie por parte dos entrevistados foi obtida por meio da seleção dos relatos que estavam de acordo com o padrão de distribuição da espécie na região (indivíduos avistados entre 100 e 1.500 m da costa e grupos de fêmeas com filhotes avistados após a arrebentação, em profundidades de 5 a 10 m) (Bottini, 2014; Groch, 2000; Payne, 1986), coloração do corpo (preta com manchas brancas e presença de calosidades na cabeça), comprimento corporal máximo (17 m) e comportamento descrito na literatura (Jefferson et al., 2015). Para as análises, foram considerados somente os relatos dos pescadores que identificaram a espécie por meio das características ora indicadas.

\section{RESULTADOS E DISCUSSÃO}

Dos 33 pescadores entrevistados, 27 (82\%) identificaram a baleia que ocorre na região como pertencente à espécie E. australis - Praia de Gamboa ( $n=10)$ e Praia de Garopaba $(n=17)-$, sendo que os relatos destes pescadores referentes ao item 3 da Tabela 1 coincidiram com as características descritas pela literatura para a espécie. Desta forma, os dados fornecidos pelos pescadores mostram-se confiáveis, ao serem comparados com a literatura sobre essa espécie. A categoria 'ecologia comportamental' foi descrita com menor grau de coincidência, provavelmente devido à complexidade em se observar a etologia de grandes baleias que pouco emergem para a superfície da água. O conhecimento empírico sobre os cetáceos adquirido por comunidades pesqueiras, por meio da prática da pesca artesanal, também foi abordado em alguns estudos ao longo da costa do Brasil. Os resultados revelaram que pescadores artesanais são capazes de identificar as espécies que se distribuem nos seus campos de pesca preferenciais (Souza \& Begossi, 2007; FreitasNetto \& Di Beneditto, 2008; Zappes et al., 2011a, 2011b, 2013a, 2013b, 2016).

\section{PERFIL DOS ENTREVISTADOS}

Os pescadores selecionados, todos do sexo masculino, apresentaram idade entre 24 e 94 anos, e a faixa etária mais frequente para as duas áreas foi entre 41 e 60 anos. Em relação ao tempo de pesca na região, as respostas foram: 14 trabalham entre 11 e 30 anos (52\%), nove há mais de 31 anos (33\%) e quatro até dez anos (15\%). Para a escolaridade, 15 entrevistados (56\%) responderam ter cursado apenas o Ensino Fundamental, oito (30\%) o Ensino Médio, dois (7\%) o Ensino Superior e dois (7\%) relataram não ter estudado. Tais características são semelhantes entre outras comunidades de pescadores artesanais localizadas no Brasil (Alencar \& Maia, 2011; Oliveira et al., 2016; Silva, C. et al., 2014; Zappes et al., 2016). Devido ao fato de a pesca artesanal permitir o sustento imediato da família, pescadores se dedicam a esta atividade, cuja rotina dificulta a dedicação aos estudos (Silva, C. et al., 2014). Dessa forma, há o abandono da escola por estes trabalhadores, o que afeta sua situação socioeconômica e, ainda, pode interferir na capacidade de organização da colônia e em associações de pescadores (Silva, M. et al., 2007). 


\section{DESCRIÇÃO DA ATIVIDADE PESQUEIRA ARTESANAL}

Os artefatos de pesca utilizados são a rede de espera/ caceio ( $n=21,88,9 \%)$, espinhel $(n=14,51,9 \%)$, rede de arrastão de praia $(n=5,18,5 \%)$, tarrafa $(n=4,14,8 \%)$, rede de arrasto/rede de camarão $(n=1,3,7 \%)$ e rede de cerco $(n=1,3,7 \%)$. Com relação às embarcações, um entrevistado (3,7\%) relatou utilizar mais de um tipo de barco (barco de arrasto, bateira e canoa). Treze pescadores $(48,1 \%)$ utilizam a bateira, que pode medir entre 7,5 e $15 \mathrm{~m}$ de comprimento e com potência do motor variando de 24 a $250 \mathrm{HP}$; canoa ( $n=4,14,8 \%)$, que varia de 6 a $10 \mathrm{~m}$ de comprimento; baleeira $(n=2,7,4 \%)$, que varia entre 9 e $14 \mathrm{~m}$ de comprimento, com motor entre $24 \mathrm{e}$ 115 HP; lancha ( $n=1,3,7 \%)$, com 9 m de comprimento e motor de $24 \mathrm{HP}$; e bote ( $n=1,3,7 \%)$, com 8 m de comprimento e motor entre 25 e $45 \mathrm{HP}$. Dois pescadores $(7,4 \%)$ relataram pescar andando pela orla da praia e cinco (18,5\%) não souberam identificar o tipo de embarcação utilizada na pescaria. Os dois pescadores que atuam na orla já tiveram experiência de prática de pesca embarcados, por isso identificaram a baleia-franca-austral como $E$. australis.

\section{CARACTERÍSTICAS DA EUBALEANA AUSTRALIS E COMPORTAMENTOS COMUNS A INDIVÍDUOS ADULTOS E INFANTES}

Os comportamentos descritos para E. australis se relacionaram àqueles exibidos durante o dia e a noite, durante a alimentação, a vocalização e frente às embarcações. A ordenação em etnocategorias considerou as classes de maturidade 'adultos e infantes' (Tabela 2), 'adultos' (Tabela 3) e 'infantes' (Tabela 4). Durante a entrevista, o mesmo pescador descreveu mais de um comportamento para cada uma destas questões, o que explica o número de relatos maior do que o número de entrevistados.

Os pescadores descreveram dez etnocategorias comportamentais comuns a ambas as classes de maturidade $(n=50)$ que se relacionaram às categorias reportadas pela literatura como pertencentes a alimentação, respiração, vocalização/eventos de

Tabela 2. Etnocategorias comportamentais comuns aos indivíduos adultos e infantes de Eubalaena australis descritas pelos pescadores do município de Garopaba, Santa Catarina, e comparação com dados da literatura. Etnoclassificação de comportamento: $A=$ exibido durante a alimentação, $V=$ exibido durante a vocalização, $E=$ frente a embarcações; classe de maturidade: $A=$ adulto, $I=$ infante; categorias comportamentais descritas na literatura: 1 = IWC/Brasil (1999), 2 = Jefferson et al. (2015), 3 = Clark (1983), 4 = Elwen \& Best (2004), 5 = Groch (2005), 6 = Lundquist (2007), 7 = Danielski (2008), 8 = Dombroski et al. (2016).

\begin{tabular}{|c|c|c|c|c|}
\hline Etnocategorias comportamentais & Etnoclassificação & $\begin{array}{l}\text { Classe de } \\
\text { maturidade }\end{array}$ & $\begin{array}{l}\text { Número de } \\
\text { relatos }\end{array}$ & $\begin{array}{l}\text { Categorias comportamentais } \\
\text { descritas na literatura }\end{array}$ \\
\hline $\begin{array}{l}\text { Segue o cardume, abre a boca e } \\
\text { come um peixe por vez }\end{array}$ & A & $\mathrm{A} / \mathrm{I}$ & 5 & Alimentação1, 2 \\
\hline Assobiar & $\vee$ & $A / I$ & 2 & Respiração ${ }^{1}$ \\
\hline Respirar & V & $\mathrm{A} / \mathrm{I}$ & 3 & \\
\hline Gritar & V & $A / I$ & 3 & $\begin{array}{l}\text { Vocalização/eventos de interação } \\
\text { (repertório acústico) })^{3,4,8}\end{array}$ \\
\hline Berrar & V & $\mathrm{A} / \mathrm{I}$ & 9 & \\
\hline Não faz nada & $E$ & $\mathrm{~A} / \mathrm{I}$ & 13 & Desinteresse, indiferença ${ }^{5,6}$ \\
\hline Afastar & $E$ & $\mathrm{~A} / \mathrm{I}$ & 6 & Afastamento ${ }^{1,5}$ \\
\hline Se o motor estiver ligado, foge & E & $\mathrm{A} / \mathrm{I}$ & 3 & \\
\hline Quando chega perto do barco, afunda & $E$ & $\mathrm{~A} / \mathrm{I}$ & 3 & Imersão e posterior emersão ${ }^{5}$ \\
\hline Se aproxima da embarcação & E & $\mathrm{A} / \mathrm{I}$ & 3 & Aproximação à embarcação 5, 6,7 \\
\hline
\end{tabular}

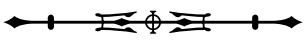


interação, desinteresse/indiferença, afastamento, imersão e posterior emersão e aproximação à embarcação (Tabela 2). A etnocategoria 'não faz nada' foi relatada com maior frequência $(n=13,26,0 \%)$ e se inseria na questão referente aos comportamentos exibidos pela baleia frente à embarcação.

Tabela 3. Etnocategorias comportamentais exclusivas de indivíduos adultos de Eubalaena australis descritas pelos pescadores do município de Garopaba, Santa Catarina, e comparação com dados da literatura. Etnoclassificação de comportamento: $\mathrm{D}=$ exibido durante o dia, $\mathrm{N}$ = exibido durante a noite, $\mathrm{A}=$ exibido durante a alimentação, $\mathrm{V}=$ exibido durante a vocalização; classe de maturidade: $\mathrm{A}=$ adulto; categorias comportamentais descritas na literatura: 1 = Groch (2000), $2=$ Danielski (2008), 3 = Thomas \& Taber (1984), $4=$ IWC/ Brasil (1999), 5 = Lyamin et al. (2007), 6 = Groch (2005), 7 = Payne (1986), 8 = Clutton-Brock (1991), 9 = Jefferson et al. (2015), 10 $=$ Clark (1983), $11=$ Ford \& Kraus (1992) e 12 = Dombroski et al. (2016).

\begin{tabular}{|c|c|c|c|c|}
\hline Etnocategorias comportamentais & Etnoclassificação & $\begin{array}{l}\text { Classe de } \\
\text { maturidade }\end{array}$ & $\begin{array}{l}\text { Número de } \\
\text { relatos }\end{array}$ & $\begin{array}{c}\text { Categorias comportamentais descritas } \\
\text { na literatura }\end{array}$ \\
\hline Nadar & $\mathrm{D} / \mathrm{N}$ & A & 6 & Deslocamento/natação", 2 \\
\hline Passear & $\mathrm{D} / \mathrm{N}$ & A & 7 & \\
\hline Viajar & N & A & 2 & Viajar (migração) $)^{3,4}$ \\
\hline Mergulhar & $\mathrm{D} / \mathrm{N}$ & A & 2 & Mergulho/não definido 4 \\
\hline Ficar parada & $\mathrm{D} / \mathrm{N}$ & A & 4 & Repouso $0^{1,2,3,5}$ \\
\hline Descansar & $\mathrm{D} / \mathrm{N}$ & A & 2 & \\
\hline Dormir & $\mathrm{D} / \mathrm{N}$ & A & 3 & Dormir/sono/deslocamento lento ${ }^{2,4}$ \\
\hline $\begin{array}{l}\text { Aconchegar com o filhote no } \\
\text { litoral }\end{array}$ & $\mathrm{D}$ & A & 1 & $\begin{array}{c}\text { Área de reprodução/área de } \\
\text { preferência', } 4,6,11\end{array}$ \\
\hline Vir procriar & $\mathrm{D}$ & A & 1 & \\
\hline Cuidar e ensinar a cria & $\mathrm{D} / \mathrm{N}$ & A & 2 & $\begin{array}{l}\text { Aprendizado e desenvolvimento do } \\
\text { filhote/imitação do comportamento das } \\
\text { mães pelo filhote }{ }^{2,3,7}\end{array}$ \\
\hline Pular & $\mathrm{D} / \mathrm{N}$ & A & 4 & $\begin{array}{c}\text { Comportamento aéreo/exibição/ } \\
\text { brincadeiras }{ }^{1,2,3,4}\end{array}$ \\
\hline Brincar & $\mathrm{D} / \mathrm{N} N$ & A & 13 & \\
\hline Colocar a nadadeira para fora & $\mathrm{D}$ & A & 1 & \\
\hline Bater a cauda & N & A & 2 & \\
\hline Comer & $\mathrm{D} / \mathrm{N}$ & A & 3 & Alimentação ${ }^{4,8}$ \\
\hline Mergulhar e dar o bote & A & A & 1 & \\
\hline $\begin{array}{l}\text { Encher a boca d'água, soltar a } \\
\text { água pela barbatana, a comida fica } \\
\text { presa na barbatana }\end{array}$ & A & A & 3 & \\
\hline Embocar o cardume & A & A & 6 & \\
\hline Gemer & $\vee$ & A & 2 & Vocalização/eventos de interação9, 10, 12 \\
\hline $\begin{array}{l}\text { Comunicar-se com o filhote e } \\
\text { com os outros }\end{array}$ & $\vee$ & A & 2 & \\
\hline Assoprar & $\vee$ & A & 1 & Respiração ${ }^{4}$ \\
\hline Suspirar & $\vee$ & A & 1 & \\
\hline Apitar & $\vee$ & A & 1 & \\
\hline Levantar a cabeça para emitir som & $\vee$ & A & 1 & \\
\hline $\begin{array}{l}\text { Jogar os animais para cima e os } \\
\text { agarrar com a boca }\end{array}$ & A & A & 1 & Não encontrado registro na literatura \\
\hline
\end{tabular}


Tabela 4. Etnocategorias comportamentais exclusivas de indivíduos infantes de Eubalaena australis descritas pelos pescadores do município de Garopaba, Santa Catarina, e comparação com dados da literatura. Etnoclassificação de comportamento: A = exibido durante a alimentação, $\mathrm{V}=$ exibido durante a vocalização; classe de maturidade: I = infante; categorias comportamentais descritas na literatura: $1=$ Clark (1983), 2 = Elwen \& Best (2004), 3 = Thomas \& Taber (1984), 4 = IWC/Brasil (1999) e 5 = Dombroski et al. (2016).

\begin{tabular}{|c|c|c|c|c|}
\hline Etnocategorias comportamentais & Etnoclassificação & $\begin{array}{l}\text { Classe de } \\
\text { maturidade }\end{array}$ & $\begin{array}{l}\text { Número de } \\
\text { relatos }\end{array}$ & $\begin{array}{l}\text { Categorias comportamentais } \\
\text { descritas na literatura }\end{array}$ \\
\hline Chama a mãe para perto & $\vee$ & I & 3 & \multirow{2}{*}{ Eventos de interação1, 2,5 } \\
\hline Pega o leite que é solto na água & A & I & 8 & \\
\hline Bate na baleia e ela solta o leite na água & A & I & 2 & \multirow{2}{*}{ Amamentação ${ }^{3,4}$} \\
\hline Suga o leite com a boca & A & I & 5 & \\
\hline
\end{tabular}

Na Península de Valdés, Argentina, foram realizados testes com embarcações de observação de baleias em geral, indicando que esses organismos podem assumir diferentes comportamentos em relação às embarcações, como evitar a aproximação, ser indiferentes à sua presença ou, em alguns casos, nadar em direção aos barcos (Lundquist, 2007). Groch (2005) aponta que o comportamento de indiferença da baleia-franca-austral em relação à embarcação pode estar relacionado com a propagação do som das embarcações nas enseadas, o que poderia inibir suas atividades.

Oito pescadores descreveram características humanas para a baleia como: mansa ( $n=3,37,5 \%)$, dócil $(n=3$, $37,5 \%)$, curiosa ( $n=1,12,5 \%)$ e sensível $(n=1,12,5 \%)$. A atribuição das características 'mansa' e 'dócil' pode se relacionar ao seu comportamento de natação lenta na superfície por longos períodos (Danielski, 2008; Groch, 2000), onde raramente executa comportamentos aéreos. A característica 'curiosa' pode se relacionar aos infantes que se aproximam da embarcação, e 'sensível', por perceber de longe a presença de embarcações. Relatos de características humanas para os cetáceos são apontados em outros estudos etnobiológicos envolvendo comunidades de pescadores artesanais no litoral brasileiro. Zappes (2007) relata a atribuição da característica 'manso' para o boto-cinza [Sotalia guianensis (van Bénéden, 1864)], na costa nordeste e sudeste, e Zappes et al. (2016) descrevem a mesma característica para a toninha (Pontoporia blainvillei Gervais \&
D'Orbigny, 1844), no Complexo Estuarino de Paranaguá, estado do Paraná, Sul do Brasil. Peterson et al. (2008) e Zappes et al. (2011a), em estudos realizados em Laguna, em Santa Catarina, e Imbé/Tramandaí, no estado do Rio Grande do Sul, registraram relatos de características antropomórficas para o golfinho-nariz-de-garrafa (Tursiops truncatus Montagu, 1821). O antropomorfismo de comportamentos de baleiafranca-austral faz com que pescadores percebam 'qualidades' nestes animais, tornando as baleias carismáticas, o que, consequentemente, pode reduzir possíveis conflitos entre a pesca artesanal e os cetáceos.

\section{COMPORTAMENTOS EXCLUSIVOS DE INDIVÍDUOS ADULTOS}

Um total de 25 etnocategorias comportamentais foi descrito para indivíduos adultos de baleia-franca-austral ( $n=72$ ). A categoria 'brincar' ( $n=13,18,1 \%)$, com a maior frequência de relatos, está relacionada aos períodos diurno e noturno e com a vocalização dos animais (Tabela 3). O termo 'brincar' é reportado na literatura para E. australis, a fim de caracterizar atividades realizadas entre mães e filhotes, em que o adulto exibe vários padrões comportamentais, desde boiar na superfície com o ventre parcialmente exposto até saltos e batidas de nadadeira caudal. Estes comportamentos podem ter significado social (aprendizado do filhote) e fisiológico (fortalecimento dos músculos para movimentos migratórios) (Groch, 2000; IWC/Brasil, 1999; Thomas \& Taber, 1984).

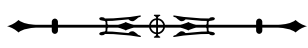


Para os comportamentos exibidos durante o período noturno, as etnocategorias 'brincar' ( $n=3,13,6 \%$ ) e 'passear' ( $n=3,13,6 \%)$ foram as mais relatadas. Durante a noite, torna-se difícil visualizar os animais e, dessa forma, ao ouvirem os ruídos produzidos pela movimentação das baleias na água, os pescadores provavelmente fazem associação com os sons produzidos durante o dia, a partir de atividades de brincadeira ou deslocamento. $\bigcirc$ etnocomportamento 'passear' está relacionado à natação do animal, que pode ser observada pelos pescadores quando a baleia emerge. Baleias-francas-austrais, durante a época de reprodução e nascimento dos filhotes, migram para águas costeiras e calmas, executando movimentos lentos na superfície, com menor gasto de energia (Elwen \& Best, 2004; Payne, 1986).

Entre os entrevistados que identificaram E. australis, 26 relataram ouvir a vocalização dos animais. Os sons foram classificados pelos pescadores em 11 etnocategorias ( $n=20)$ e o comportamento mais frequente foi 'berrar' ( $n=6,30 \%$ ). Além disso, foram identificados os seguintes comportamentos de vocalização: 'levantar a cabeça para emitir som' ( $n=2)$, 'gritar' $(n=2)$, 'assoprar' $(n=1)$, 'suspirar' ( $n=1)$, 'apitar' $(n=1)$, 'assobiar' $(n=1)$ e 'respirar' $(n=1)$. Vocalização de baleias tem sido amplamente estudada nos últimos anos (Dombroski et al., 2016, 2017; Hofmeyr-Juritz, 2010; Parks \& Tyack, 2005; Parks et al., 2011; Tyack \& Clark, 2000), entretanto, devido à dificuldade de se observar o comportamento do animal no momento em que vocaliza, pouco se sabe sobre a relação desses sons com suas atividades (Dombroski et al., 2016, 2017; Parks et al., 2011).

Quanto aos eventos 'levantar a cabeça para emitir som', 'assoprar', 'suspirar', 'apitar', 'assobiar', 'respirar' e 'gritar', estes parecem estar mais associados com a atividade respiratória da espécie do que com a comunicação propriamente dita. Ao emergir para respirar, E. australis produz um 'esguicho' ou 'borrifo' em formato de ' $\mathrm{V}$ ', que pode atingir de $5 \mathrm{a} 8 \mathrm{~m}$ de atura, resultante do ar quente expelido e da vaporização de gotículas de água contidas nos dois orifícios respiratórios (Jefferson et al., 2015). O som provocado pelo ar expelido pode ser ouvido a centenas de metros, o que justifica os relatos desses eventos serem descritos pelos pescadores como pertencentes à vocalização.

Para a questão relacionada com a alimentação, foram descritas quatro etnocategorias $(n=11)$, entre as quais 'embocar o cardume' ( $n=6,54,5 \%$ ) foi a mais frequente. A alimentação das baleias-francas-austrais nas regiões subpolares é constituída de zooplânctons, principalmente de pequenos copépodes e eufausídeos (Goodall \& Galeazzi, 1986; IWC/Brasil, 1999; Jefferson et al., 2015; Moore et al., 1999). Entretanto, não há registro de atividade alimentar da espécie, ao migrar para áreas de baixas latitudes relacionadas à reprodução, como a APA da Baleia Franca. O fato de os pescadores de Garopaba e Gamboa relatarem a ocorrência da alimentação da baleia-franca-austral para a costa do estado de Santa Catarina pode ser justificado pela observação do comportamento de superfície, na qual a baleia abre a boca. Este comportamento, apesar de ser pouco comum em áreas costeiras de reprodução, está relacionado com a termorregulação corporal, e não com a alimentação (Ford \& Kraus, 1992).

\section{COMPORTAMENTOS EXCLUSIVOS DE INDIVÍDUOS INFANTES}

Quando questionados sobre os indivíduos infantes, os pescadores relataram quatro etnocategorias comportamentais $(n=18)$, duas relacionadas à alimentação/ amamentação ( $n=15,83,3 \%)$ e duas à vocalização com eventos de interação $(n=3,16,7 \%)$ (Tabela 4). Para alimentação, foi descrito principalmente 'pegar o leite que é solto na água' ( $n=8,53,3 \%)$ e, para vocalização, 'chamar a mãe para perto' ( $n=3,100 \%)$.

Todas as etnocategorias referentes à alimentação do filhote se relacionavam à amamentação. $O$ comportamento 'pegar o leite que é solto na água' pode ter sido presenciado pelos pescadores durante a 
pesca, já que a APA da Baleia Franca é uma das áreas de amamentação da espécie. Durante a amamentação, o leite é ejetado, por músculos da região mamária, direto das glândulas mamárias da mãe para dentro da boca do filhote. Este comportamento é repetido ao longo de todo o dia, supondo que o filhote mergulha a curtos intervalos para mamar (Thomas \& Taber, 1984). Os pescadores identificam a baleia-franca-austral como pertencente ao grupo dos mamíferos, podendo compará-la ao comportamento de amamentação em humanos.

Em relação à vocalização entre infantes e adultos, sabe-se que pares de mães e filhotes utilizam sons na faixa entre 100 e $200 \mathrm{~Hz}$ para manter contato, mesmo em curtas distâncias (Clark, 1983). Além disso, os filhotes passam a maior parte do tempo das primeiras semanas de vida na companhia das fêmeas, em áreas costeiras e de pouca profundidade (Elwen \& Best, 2004). A proximidade da linha de costa permite aos pescadores visualizar grupos formados por fêmeas e filhotes. Por meio da prática diária da atividade de pesca artesanal na APA da Baleia Franca, os entrevistados percebem que o contato do infante com a mãe é intenso. Estas observações permitiram a elaboração de um conhecimento ecológico local sobre o comportamento de vocalização entre mãe e filhote, no qual a emissão de sons pelo último tem a função 'chamar a mãe'.

Neste estudo, foi possível verificar semelhança entre os conhecimentos dos pescadores e a literatura em relação ao comportamento da baleia-franca-austral. Assim, o conhecimento etnoecológico se confirma como uma ferramenta de geração de dados sobre a população de E. australis que ocorre na APA da Baleia Franca. Desta forma, a compreensão e a valorização deste saber local de pescadores artesanais sobre cetáceos potencializam o incremento de ações de conservação ao longo das áreas de distribuição das espécies (Zappes et al., 2013b). Ainda, devido ao aumento da população de baleia-franca-austral que migra anualmente para o litoral do estado de Santa Catarina, é importante a continuidade de estudos voltados ao conhecimento ecológico local, auxiliando a prevenção de possíveis impactos antrópicos sobre a espécie na região. A aproximação de órgãos de pesquisa com a comunidade pesqueira poderá auxiliar na elaboração de estratégias para o manejo da pesca artesanal na região e no estabelecimento de diretrizes para planos de gestão comunitária nessa APA, visando à manutenção da população da espécie no sítio de reprodução do Atlântico Sul e a continuidade da atividade pesqueira.

\section{AGRADECIMENTOS}

Agradecemos ao presidente do Sindicato dos Pescadores de Garopaba (Z-12), a todos os pescadores entrevistados, a S. C. Moreira, pela elaboração do mapa, aos pesquisadores do Instituto Baleia Franca, em especial a M. Pontalti e C.V. da Silva, pelo apoio logístico durante a etapa de campo, a L. Lodi, A. Zerbini e A. Andriolo (Projeto Monitoramento de Baleias por Satélite), pelas imagens da prancha. Dois revisores anônimos contribuíram com comentários e sugestões para o manuscrito. CAZ agradece à Fundação Carlos Chagas Filho de Amparo à Pesquisa do Estado do Rio de Janeiro (FAPERJ E-26/203.202/2016; E-26/202.789/2019) e ao Conselho Nacional de Desenvolvimento Científico e Tecnológico (CNPq 400053/2016-0) e APMDB, à FAPERJ (E-26/202.770/2017 e E-26/210.064/2018) e ao CNPq (301.259/2017-8), pelo fomento e pela bolsa de pesquisa.

\section{REFERÊNCIAS}

ALENCAR, C. A. G. \& L. P. MAIA, 2011. Perfil socioeconômico dos pescadores brasileiros. Arquivos de Ciência do Mar 44(3): 12-19.

BAILEY, K. D., 1994. Methods of social research: 1-588. The Free Press/Macmillan Publishers, New York.

BEGOSSI, A., 2001. Mapping spots: fishing areas or territories among islanders of the Atlantic Forest (Brazil). Regional Environment Change 2(1): 1-12. DOI: https://doi.org/10.1007/s101130100022

BERKES, F., 1999. Sacred ecology: traditional ecological knowledge and resource management: 1-209. Taylor and Francis, Philadelphia.

BERNARD, H. R., 2000. Social research methods: qualitative and quantitative approaches: 1-412. Sage Publications, Thousand Oaks, California.

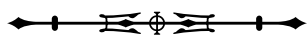


BIERNACKI, P. \& D. WALDORF, 1981. Snowball sampling: problems and techniques of chain referral sampling. Sociological Methods and Research 10(2): 141-163. DOI: https://doi. org/10.1177/004912418101000205

BOTTINI, S. F., 2014. Distribuição espacial e padrões de movimento da baleia-franca-austral (Eubalaena australis) em Torres, Rio Grande do Sul, Brasil. Dissertação (Mestrado em Biologia Animal) - Universidade Federal do Rio Grande do Sul, Porto Alegre.

BRASIL, 2015. Lei n 13.123, de 20 de maio de 2015. Regulamenta o inciso II do $\S 1^{\circ}$ e o $\S 4^{\circ}$ do art. 225 da Constituição Federal, o Artigo 1, a alínea j do Artigo 8, a alínea c do Artigo 10, o Artigo 15 e os $\S \S 3^{\circ}$ e $4^{\circ}$ do Artigo 16 da Convenção sobre Diversidade Biológica, promulgada pelo Decreto $n^{\circ} 2.519$, de 16 de março de 1998; dispõe sobre o acesso ao patrimônio genético, sobre a proteção e o acesso ao conhecimento tradicional associado e sobre a repartição de benefícios para conservação e uso sustentável da biodiversidade; revoga a Medida Provisória n 2.186-16, de 23 de agosto de 2001; e dá outras providências. Diário Oficial da União. Disponível em: http://www.planalto.gov.br/ccivil_03/_ato20152018/2015/lei/13123.htm. Acesso em: 15 outubro 2017.

BUELONI, F. S., 2012. Mudanças temporais na utilização na baleia franca, Eubalaena australis, pelas comunidades locais dos municípios de Imbituba e Garopaba, litoral sul de Santa Catarina, Brasil. Dissertação (Mestrado em Ecologia) - Universidade Federal de Santa Catarina, Florianópolis.

CAPELlesso, A. J. \& A. A. CAZELLA, 2011. Pesca artesanal entre crise econômica e problemas socioambientais: estudo de caso nos municípios de Garopaba e Imbituba (SC). Ambiente \& Sociedade 14(2): 15-33. DOI: http://dx.doi.org/10.1590/S1414$753 \times 2011000200003$

CLARK, C. W., 1983. Acoustic communication and behavior of the southern right whale (Eubalaena australis). Communication and Behavior of Whales 17(2): 163-198.

CLAUZET, M., M. RAMIRES \& W. BARRELLA, 2005. Pesca artesanal e conhecimento local de duas populações caiçaras (Enseada do Mar Virado e Barra do una) no litoral de São Paulo, Brasil. Multiciência 4: $1-22$

CLUTTON-BROCK, T. H., 1991. The evolution of parental care: 1-333. Princeton University Press, New Jersey.

COSTA-NETO, E. M., 2000. Sustainable development and traditional knowledge: a case study in a Brazilian artisanal fishermen's community. Sustainable Development 8(2): 89-95. DOI: https://doi.org/10.1002/(SICI)1099-1719(200005)8:2<89::AIDSD130>3.0.CO;2-S

DANIELSKI, M. L., 2008. Comportamentos de mães e filhotes de baleia-franca-austral, Eubalaena australis (Desmoulins, 1822) em Santa Catarina, Brasil. Dissertação (Mestrado em Ecologia) Universidade Federal de Juiz de Fora, Juiz de Fora.
DIEGUES, A. C., 2000. Etnoconservação da natureza: enfoques alternativos. In: A. C. DIEGUES (Org.): Etnoconservação: novos rumos para a proteção da natureza nos trópicos: 1-46. NUPAUB/ USP, São Paulo.

DIEGUES, A. C., 2002. Sea Tenure, traditional knowledge and management among Brazilian artisanal fishermen: 1-58. NUPAUB/USP, São Paulo.

DOMBROSKI, J. R. G., S. E. PARKS, K. R. GROCH, P. A. C. FLORES \& R. S. SOUSA-LIMA, 2016. Vocalizations produced by southern right whale (Eubalaena australis) mother-calf pairs in a calving ground off Brazil. Journal of the Acoustical Society of America 140(3): 1850-1857. DOI: https://doi.org/10.1121/1.4962231

DOMBROSKI, J. R. G., S. E. PARKS, K. R. GROCH, P. A. C. FLORES \& R. S. SOUSA-LIMA, 2017. Upcall production by southern right whale (Eubalaena australis) mother-calf pairs may be independent of diel period in a nursery area. Marine Mammal Science 33(2): 669-677. DOI: https://doi.org/10.1111/mms.12382

ELWEN, S. H. \& P. B. BEST, 2004. Female southern right whales Eubalaena australis: are there reproductive benefits associated with their coastal distribution off South Africa? Marine Ecology Progress Series 269: 289-295. DOI: https://doi.org/10.3354/meps269289

FARIAS, G. B. \& Â. G. C., ALVES, 2007. Aspectos históricos e conceituais da etnoornitologia. Biotemas 20(1): 91-100.

FORD, T. \& S. KRAUS, 1992. A rete in the right whale. Nature 359(680): 5-24. DOI: https://doi.org/10.1038/359680a0

FREITAS-NETTO, R. F. \& A. P. M. DI BENEDITTO, 2008. Interactions between fisheries and cetaceans in Espírito Santo State coast, southeastern Brazil. Revista Brasileira de Zoociências 10(1): 55-63.

GOODALL, R. M. P. \& A. R. GALEAZZI, 1986. Recent sightings and strandings of southern right whales off Subantarctic South America and the Antarctic Peninsula. Report of the International Whaling Commission 10: 173-176.

GROCH, K. R., 2000. Ocupação preferencial de áreas de concentração pela baleia franca austral, Eubalaena australis (Desmoulins, 1822), Cetacea, Mysticeti, no litoral sul do Brasil. Dissertação. (Mestrado em Biologia Animal) - Universidade Federal do Rio Grande do Sul, Porto Alegre.

GROCH, K. R., 2005. Biologia populacional e ecologia comportamental da baleia franca austral, Eubalaena australis (Desmoulins, 1822), Cetacea, Mysticeti, no litoral sul do Brasil. Tese (Doutorado em Biologia Animal) - Universidade Federal do Rio Grande do Sul, Porto Alegre.

GROCH, K. R., J. T. J. R. PALAZZO, P. A. C. FLORES, F. R. ADLER \& M. E. FABIAN, 2005. Recent rapid increases in the right whale (Eubalaena australis) population off southern Brazil. Latin American Journal of Aquatic Mammals 4(1): 41-47. DOI: http://dx.doi. org/10.5597/lajam00068

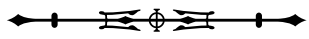


HOFMEYR-JURITZ, L. H., 2010. The nature and rate of vocalization by southern right whales (Eubalaena australis), and the evidence for individually distinctive calls. Tese (Doutorado em Filosofia) - Universidade de Pretoria, Pretoria.

HUNTINGTON, H. O., P. K. BROWN-SCHWALENBERG, K. J. FROST, M. E. FERNANDEZ-GIMENEZ, D. W. NORTON \& D. H. ROSENBERG, 2002. Observations on the workshop as a means of improving communication between holders of traditional and scientific knowledge. Environmental Management 30: 778-792. DOI: https://doi.org/10.1007/ s00267-002-2749-9

IWC/BRASIL, 1999. Plano de ação para conservação da baleia franca, Eubalaena australis, no estado de Santa Catarina: 1-59. IWC, Florianópolis.

JEFFERSON, T. A., M. A. WEBBER \& R. L. PITMAN, 2015. Marine mammals of the World: a comprehensive guide to their identification: 2 ed.: 1-616. Academic Press, San Diego. DOI: https://doi.org/10.1016/C2012-0-06919-0

KVALE, S., 1996. Interviews: an introduction to qualitative research interviewing: 1-353. Sage Publications, Thousand Oaks, London.

LIBRETT, M. \& D. PERRONE, 2010. Apples and oranges: ethnography and the IRB. Qualitative Research 10(6): 729-747. DOI: https://doi.org/10.1177/1468794110380548

LUNDQUIST, D. J., 2007. Behavior and movement of southern right whales: effects of boats and swimmers. Dissertação (Mestrado em Ciências) - Texas A\&M University, College Station.

LYAMIN, O., J. PRYASLOVA, P. KOSENKO \& J. SIEGEL, 2007. Behavioral aspects of sleep in bottlenose dolphin mothers and their calves. Physiology \& Behavior 92(4): 725-733. DOI: http:// dx.doi.org/10.1016/j.physbeh.2007.05.064

MASON, M., 2010. Sample size and saturation in PhD studies using qualitative interviews. Forum for Qualitative Social Research 11(3): 1-19. DOI: http://dx.doi.org/10.17169/fqs11.3.1428

MIRANDA, T. M., M. C. M. AMOROZO, J. S. GOVONE \& M. M. DANIELA, 2007. The influence of visual stimuli in ethnobotanical data collection using the listing task method. Field Methods 19(1): 76-86. DOI: https://doi.org/10.1177/1525822X06295987

MOORE, M. J., S. D. BERROW, B. A. JENSEN, P. CARR, R. SEARS, V. J. ROWNTREE, R. PAYNE \& P. K. HAMILTON, 1999. Relative abundance of large whales around South Georgia (1979-1998). Marine Mammal Science 15(4): 1287-1302. DOI: https://doi. org/10.1111/j.1748-7692.1999.tb00891.x

MORSE, J. M., 1994. Designing funded qualitative research. In: N. K. DENZIN \& Y. S. LINCOLN (Ed.): Handbook of qualitative research: 220-235. Sage Publications, Thousand Oaks.
OLIVEIRA, P. C., A. P. M. DI BENEDITTO, E. M. R. BULHÕES \& C. A. ZAPPES, 2016. Artisanal fishery versus port activity in southern Brazil. Ocean \& Coastal and Management 129: 49-57. DOI: http:// dx.doi.org/10.1016/j.ocecoaman.2016.05.005

PAES, R. S. \& C. A. ZAPPES, 2016. Agricultura familiar no norte do estado do Rio de Janeiro: identificação de manejo tradicional. Sociedade e Natureza 28(3): 385-395. DOI: https://doi. org/10.1590/1982-451320160305

PARKS, S. E. \& P. L. TYACK, 2005. Sound production by North Atlantic right whales (Eubalaena glacialis) in surface active groups. Journal of the Acoustical Society of America 117(5): 3297-3306. DOI: https://doi.org/10.1121/1.1882946

PARKS, S. E., A. SEARBY, A. CÉLÉRIER, M. P. JOHNSON, D. P. NOWACEK \& P. L. TYACK, 2011. Sound production behavior of individual North Atlantic right whales: implications for passive acoustic monitoring. Endangered Species Research 15: 63-76. DOI: https:// doi.org/10.3354/esr00368

PAYNE, R., 1986. Long term behavioral studies of the southern right whale (Eubalaena australis). Report of the International Whaling Commission Special 10: 161-167.

PETERSON, D., N. HANAZAKI \& P. C. SIMÕES-LOPES, 2008. Natural resource appropriation in cooperative artisanal fishing between fishermen and dolphins (Tursiops truncatus) in Laguna, Brazil. Ocean \& Coastal and Management 51(6): 469-475. DOI: https://doi.org/10.1016/j.ocecoaman.2008.04.003

REBOUÇAS, G. N., 2008. Regimes de apropriação, usos e conflitos de uso do espaço marinho e seus recursos: estudo de caso no município de Garopaba, litoral centro-sul de Santa Catarina. Dissertação (Mestrado em Sistemas Costeiros e Oceânicos) Universidade Federal do Paraná, Pontal do Paraná.

ROCHA, D. \& B. DEUSDARÁ, 2005. Análise de conteúdo e análise do discurso: aproximações e afastamentos na (re)construção de uma trajetória. Alea: Estudos Neolatinos 7(2): 305-322. DOI: http:// dx.doi.org/10.1590/S1517-106×2005000200010

ROUÉ, M., 2000. Novas perspectivas em Etnoecologia: saberes tradicionais e gestão dos recursos naturais. In: A. C. DIEGUES (Org.): Etnoconservação: novos rumos para a proteção da natureza nos trópicos: 67-80. NUPAUB/USP, São Paulo.

SANCHES, R. A., 2004. Caiçaras e a Estação Ecológica de JuréiaItatins. Histórico de ocupação no contexto político, econômico, social e ambiental do Vale do Ribeira. In: O. A. V. MARQUES \& W. DULEBA (Org.): Estação Ecológica Juréia-Itatins: ambiente físico, flora e fauna: 349-359. Holos Editora, Ribeirão Preto.

SANTOS, L. M. K., 2006. Dinâmica da pesca artesanal em duas comunidades ribeirinhas tradicionais do rio Cuiabá: uma abordagem ecológica. Dissertação (Mestrado em Ecologia e Conservação da Biodiversidade) - Universidade Federal de Mato Grosso, Cuiabá. 
SCHENSUL, S. L., J. J. SCHENSUL \& M. D. LECOMPTE, 1999. Essential ethnographic methods: observations, interviews and questionnaires: 1-293. Altamira Press, Walnut Creek.

SILVA, C. V., S. C. MOREIRA, C. A. ZAPPES \& A. P. M. DI BENEDITTO, 2014. Pesca artesanal e cetáceos que ocorrem no litoral leste do Rio de Janeiro: uma abordagem etnoecológica para verificar a existência de manejo tradicional. Boletim do Instituto de Pesca 40(4): 521-539.

SILVA, M. C., A. S. OLIVEIRA \& G. Q. NUNES, 2007. Caracterização socioeconômica da pesca artesanal no município de Conceição do Araguaia, estado do Pará. Amazônia: Ciência \& Desenvolvimento 2(4): 37-51.

SOUZA, S. P. \& A. BEGOSSI, 2007. Whales, dolphins or fishes? The ethnotaxonomy of cetaceans in São Sebastião, Brazil. Journal of Ethnobiology and Ethnomedicine 3(9). DOI: http://dx.doi. org/10.1186/1746-4269-3-9

THIRY-CHERQUES, H. R., 2009. Saturação em pesquisa qualitativa: estimativa empírica de dimensionamento. Revista Brasileira de Pesquisas em Marketing (PMKT) 3: 20-27.

THOMAS, P. O. \& S. TABER, 1984. Mother-infant interaction and behavioral development in southern right whales, Eubalaena australis. Behaviour 88(1-2): 42-60.

TYACK, P. L. \& C. W. CLARK, 2000. Communication and acoustic behavior of dolphins and whales. In: W. W. L. AU, A. N. POPPER \& R. R. FAY (Ed.): Hearing by whales and dolphins: 156-224. SpringerVerlag, New York.

ZAPPES, C. A., 2007. Estudo etnobiológico comparativo do conhecimento popular de pescadores em diferentes regiões do litoral brasileiro e implicações para a conservação do boto-cinza Sotalia guianensis (van Bénéden, 1864) (Cetacea, Delphinidae). Dissertação (Mestrado em Comportamento e Biologia Animal) Universidade Federal de Juiz de Fora, Juiz de Fora.
ZAPPES, C. A., A. ANDRIOLO, P. C. SIMÕES-LOPES \& A. P. M. DI BENEDITTO, 2011a. 'Human-dolphin (Tursiops truncatus Montagu, 1821) cooperative fishery' and its influence on cast net fishing activities in Barra de Imbé/Tramandaí, Southern Brazil. Ocean \& Coastal Management 54(5): 427-432. DOI: https://doi.org/10.1016/j. ocecoaman.2011.02.003

ZAPPES, C. A., C. E. N. GATTS, L. LODI, A. ANDRIOLO \& A. P. M. DI BENEDITTO, 2011b. Interações entre o golfinho-narizde-garrafa (Tursiops truncatus, Montagu, 1821) e a pesca artesanal no Arquipélago das Cagarras e áreas adjacentes, RJ. Sitientibus, Série Ciências Biológicas 11(1): 24-30.

ZAPPES, C. A., L. C. P. S. ALVES, C. V. SILVA, A. F. AZEVEDO, A. P. M. DI BENEDITTO \& A. ANDRIOLO, 2013a. Accidents between artisanal fisheries and cetaceans on the Brazilian coast and Central Amazon: proposals for integrated management. Ocean \& Coastal Management 85(A): 46-57. DOI: https://doi.org/10.1016/j. ocecoaman.2013.09.004

ZAPPES, C. A., C. V. SILVA, M. PONTALTI, M. L. DANIELSKI \& A. P. M. DI BENEDITTO, 2013b. The conflict between the southern right whale and coastal fisheries on the southern coast of Brazil. Marine Policy 38: 428-437. DOI: https://doi.org/10.1016/j. marpol.2012.07.003

ZAPPES, C. A., R. M. GAMA, C. DOMIT, C. E. N. GATTS \& A. P. M. DI BENEDITTO, 2016. Artisanal fishing and the franciscana (Pontoporia blainvillei) in Southern Brazil: ethnoecology from the fishing practice. Journal of the Marine Biological Association of the United Kingdom 98(4): 867-877. DOI: https://doi.org/10.1017/ S0025315416001788

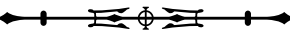


Anexo 1. Questionário semiestruturado aplicado aos pescadores.

\section{Perfil dos entrevistados}

Local

Data

Nome do entrevistado

Idade do entrevistado

Sexo do entrevistado

Escolaridade

Tempo de pesca

Tempo de pesca na região estudada

Autonomia/mar (dias/embarque)

\section{Descrição da atividade pesqueira artesanal}

Tipo de embarcação e capacidade

Potência do motor

Comprimento/embarcação

Horário da pesca

Local da pesca

\section{Artefatos}

Tipo de rede

Tamanho da malha

Altura e comprimento

Profundidade em que é armada na água

Época do ano em que utiliza o artefato

Horário em que é armada na água

Horário em que é retirada da água

Espécies-alvo

\section{Espinhel}

Extensão/comprimento

Profundidade em que é lançado

Época do ano em que utiliza o artefato

Horário em que é lançado na água

Horário em que é retirado da água

Espécies-alvo

Informações sobre outros artefatos

\section{Características de Eubalaena australis}

Quais animais não alvo da pesca são possíveis de serem observados no mar?

Tem mais de um 'tipo' (espécie) de baleia? （ ) Sim （）Não ( ) Não respondeu

Qual baleia aparece mais?

Descreva as características desta baleia (comprimento corporal, coloração e área de ocorrência)

Qual o alimento desta baleia?

Como ela se alimenta? Como é o comportamento de alimentação?

Em qual época do ano esta baleia aparece na região?
As baleias emitem algum som?

( ) $\operatorname{Sim}($ ) Não ( ) Não respondeu

Qual o comportamento da baleia quando está emitindo som?

São avistados filhotes? ( ) Sim ( ) Não ( ) Não respondeu

Qual a época do ano em que o filhote aparece na região?

Como é o filhote?

Qual é o alimento do filhote?

Como o filhote se alimenta? Como é seu comportamento de alimentação?

Os filhotes emitem algum tipo de som?

( ) $\operatorname{Sim}$ ( ) Não ( ) Não respondeu

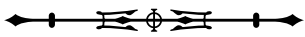


Anexo 1.

(Conclusão)

Qual o comportamento do filhote quando emite som?

Baleias são avistadas:

( ) Sozinhas ( ) Grupos ( ) Não respondeu

Se em grupos, quantas baleias são avistadas?

Onde na região as baleias são mais avistadas?

O que a baleia faz quando o barco se aproxima?

( ) Mar aberto ( ) Praia ( ) Não respondeu

O que a baleia mais faz durante o dia (comportamentos)?

O que a baleia mais faz durante a noite (comportamentos)?

O que a baleia faz quando fica próxima aos artefatos (principalmente rede)?

Porque exibem este comportamento?

\section{Utilização da prancha}

Qual destes animais é a baleia?

Qual destas você avista?

$\begin{array}{lllll}\text { ( ) } \mathrm{A} & \text { ( ) B } & \text { ( ) C } & \text { ( ) D } & \text { ( ) Não respondeu }\end{array}$

Qual o nome de cada uma dessas baleias da prancha?

$\begin{array}{lllll}\text { ( ) A } & \text { ( ) B } & \text { ( )C } & \text { ( ) D } & \text { ( ) Não respondeu }\end{array}$


Anexo 2. Fotos originais utilizadas na prancha ilustrativa apresentada aos pescadores entrevistados. Espécies: A) orca (Orcinus orca), B) baleia-franca-austral (Eubalaena australis), C) baleia-de-bryde (Balaenoptera edeni) e D) baleia-jubarte (Megaptera novaeangliae).
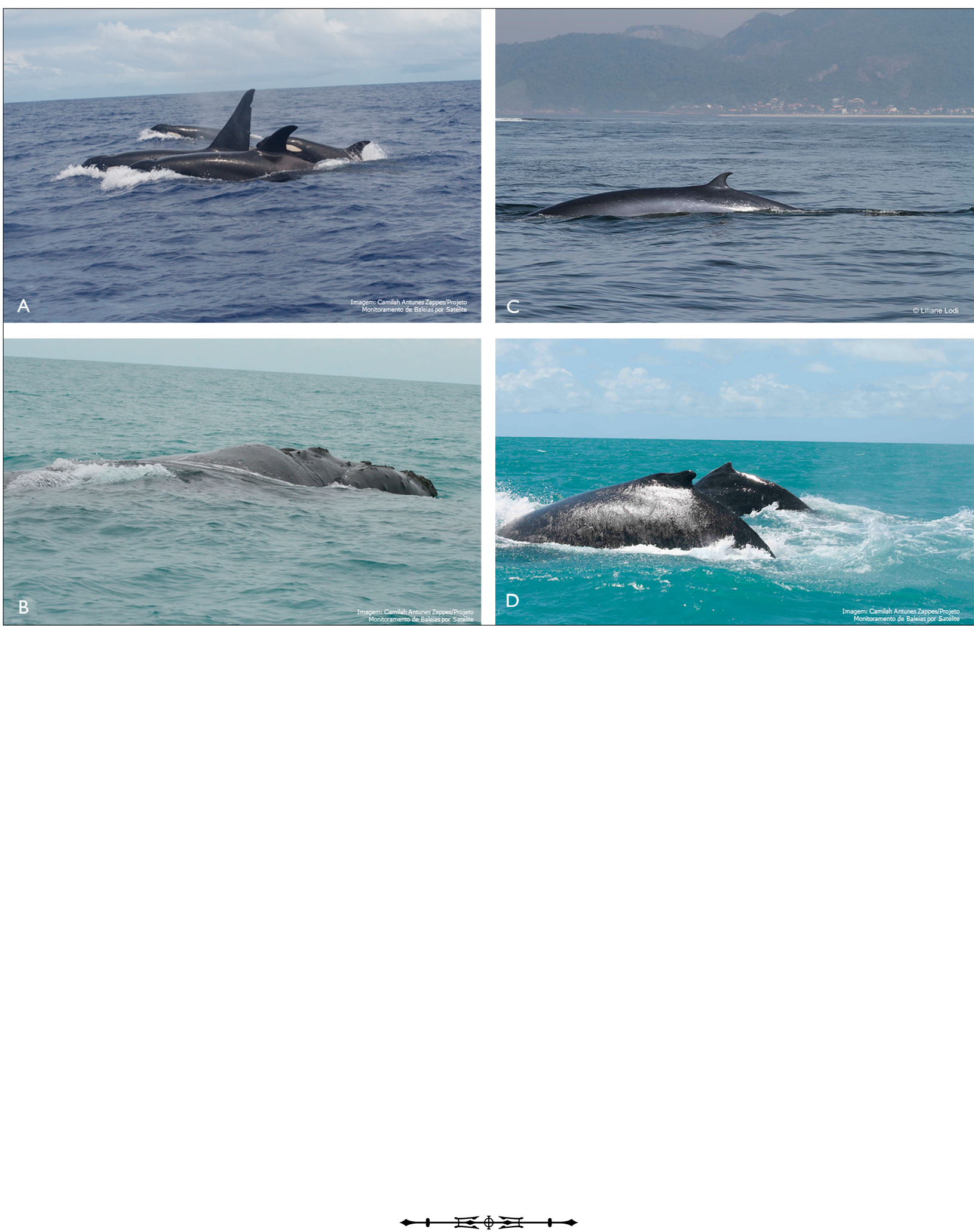